\title{
XII. Reduction of the four forms of $\omega$ in Jacobi's general transformation of an elliptic function to one form only
}

\section{Rev. Brice Bronwin}

To cite this article: Rev. Brice Bronwin (1845) XII. Reduction of the four forms of $\omega$ in Jacobi's general transformation of an elliptic function to one form only, Philosophical Magazine Series 3, 27:177, 42-46, DOI: 10.1080/14786444508645224

To link to this article: http://dx.doi.org/10.1080/14786444508645224

Published online: 30 Apr 2009.

Submit your article to this journal $\sqsubset$

山 Article views: 3

$Q$

View related articles 
period only amounted to $\frac{2 \cdot 4}{10}$. The temperature had reached its greatest of heat on the 24 th at $4^{\mathrm{h}}$ p.m., viz. $+70^{\circ} \cdot 1$, at which time the hygrometer was $+59^{\circ} \cdot 2$; the wind nearly calm and veering to $E$., and in the evening to $S$. The barometer had been gradually falling from the morning of the 21st, and at $6^{\mathrm{h}}$ p.m. a heavy thunder-storm passed over High Field House from the S. moving to the N.; the lightning was most vivid at $6^{\mathrm{h}} 1 \mathrm{~g}^{\mathrm{m}}$ p.m. In the morning a faint solar halo was formed, and in the evening an arc of a solar iris, very sensibly prismatic, was visible. At $9^{\mathrm{h}} 35^{\mathrm{m}}$ (mean time), the night, which was very dark, suddenly became light as day, and the objects near and distant were visible as plainly as in broad daylight: immediately a magnificent meteor, of a blue colour, was seen traversing the interval from the zenith, through the stars 21, 30, 40 and 41 of the constellation of Leo Minor, and the stars $95,96, x, 59, \tau$ and 75 of the constellation of Leo Major (a distance of $30^{\circ}$ ), which it accomplished in little less than three seconds of time: it exploded very near the star $\phi$, Leonis Majoris, and, after falling in small fragments of light for the space of $1^{\circ}$, became suddenly extinguished. Its apparent size was very nearly equal to the disc of the moon, and perfectly round in form; but its brilliancy very far surpassed that luminary, and its intensity could not possibly have been less than three times as light as our satellite. No train of light was left behind the meteor, as is seen with the caudate meteors. It appeared of no considerable height above the surface of our earth. There were no clouds visible at the time; but a few cumuli appeared soon after, and the moon rose of a red colour.

Should any one have noticed this phænomenon in the azimuth of the meteor, a comparison of remarks would prove both interesting and important, for the height of the meteor above the surface of our earth might be ascertained.

XII. Reduction of the Four Forms of $\omega$ in Jacobi's General Transformation of an Elliptic Function to one form only. By the Rev. Brice Bronwin*.

THE constants in Jacobi's transformation of an elliptic 1 function are all expressed by the two series of quantities-

$$
\begin{aligned}
& \sin ^{2} a m(4 \omega), \sin ^{2} a m(8 \omega) \ldots \ldots \sin ^{2} a m(2 n-2) \omega, \\
& \sin ^{2} \operatorname{co} a m(4 \omega), \sin ^{2} \operatorname{coam}(8 \omega) \ldots \ldots \sin ^{2} \operatorname{coam}(2 n-2) \omega .
\end{aligned}
$$

* Communicated by the Author. 
But $\quad \sin a m(2 n \omega-2 \omega)=\mp \sin a m(2 \omega)$,

and

$$
\sin a m(2 n \omega-6 \omega)=\mp \sin a m(6 \omega), \& c \text {. }
$$

$\sin \operatorname{coam}(2 n \omega-2 \omega)=\sin a m(\mathrm{~K}+2 \omega-2 n \omega)$

$= \pm \sin a m(\mathrm{~K}+2 \omega)=\mp \sin a m(-\mathrm{K}-2 \omega)$

$=\mp \sin a m(\mathrm{~K}-2 \omega-2 \mathrm{~K})= \pm \sin a m(\mathrm{~K}-2 \omega)$

$= \pm \sin \operatorname{coam}(2 \omega), \sin \operatorname{coam}(2 n \omega-6 \omega)= \pm \sin \operatorname{coam}(6 \omega)$, \&c.

These constants therefore may be all expressed by the two series

$$
\begin{aligned}
& \sin ^{2} \text { am }(2 \omega), \sin ^{2} a m(4 \omega) \ldots \ldots \sin ^{2} \text { am }(n-1) \omega, \\
& \sin ^{2} \operatorname{co} \text { an }(2 \omega), \sin ^{2} \operatorname{co~am~}(4 \omega) \ldots \ldots \sin ^{2} \operatorname{co} \text { am }(n-1) \omega .
\end{aligned}
$$

Consequently the $4 \omega$ in this theory may be everywhere replaced by $2 \omega$; and this reduction Jacobi has himself partially made.

But

$$
\begin{gathered}
\sin ^{2} \operatorname{coam}(2 \omega)=\frac{\cos ^{2} a m(2 \omega)}{\Delta^{2} a m(2 \omega)}=\frac{1-\sin ^{2} a m(2 \omega)}{1-k^{2} \sin ^{2} a m(2 \omega)}, \\
\sin ^{2} \operatorname{coam}(4 \omega)=\frac{1-\sin ^{2} a m(4 \omega)}{1-k^{2} \sin ^{2} a m(4 \omega)}, \text { \&c. }
\end{gathered}
$$

And $\sin ^{2} a m(4 \omega), \sin ^{2} a m(6 \omega)$, \&c. may all be expressed by functions of $\sin ^{2} a m(2 \omega)$. Therefore all the constants in this theory may be expressed by functions of $\sin ^{2} a m(2 \omega)$.

Now $\sin ^{2} a m(2 \omega)=\sin ^{2} a m(2 \omega-2 \mathrm{~K})=\sin ^{2} a m\left(2 \omega^{\prime}\right)$, if $\omega^{\prime}=\omega-\mathrm{K}$. Let

$$
\omega=\frac{2 r \mathrm{~K}+2 r^{\prime} \mathrm{K}^{\prime} \sqrt{-1}}{n} ;
$$

and make $n=2 p-1, r-p=r_{1}$; we have

$$
\omega^{\prime}=\frac{(2 r-2 p+1) \mathrm{K}+2 r^{\prime} \mathrm{K}^{\prime} \sqrt{-1}}{n}=\frac{\left(2 r_{1}+1\right) \mathrm{K}+2 r^{\prime} \mathrm{K}^{\prime} \sqrt{-1}}{n} \text {. }
$$

Hence the second form of $\omega$ reduces to the first.

Again,

$\sin ^{2} a m(2 \omega)=\sin ^{2} a m\left(2 \omega-2 \mathrm{~K}-2 \mathrm{~K}^{\prime} \sqrt{-1}\right)=\sin ^{2} a m\left(2 \omega^{\prime}\right)$,

if $w^{\prime}=\omega-K-K^{\prime} \sqrt{-1}$. Let

$$
\omega=\frac{2 r \mathrm{~K}+\left(2 r^{\prime}+1\right) \mathrm{K}^{\prime} \sqrt{-1}}{n},
$$

and make $r_{1}^{\prime}=r^{\prime}-p+1, r_{1}$ and $p$ remaining as before; we have in this case,

$$
\begin{gathered}
\omega^{\prime}=\frac{(2 r-2 p+1) K+\left(2 r^{\prime}-2 p+2\right) K^{\prime} \sqrt{-1}}{n} \\
=\frac{\left(2 r_{1}+1\right) K+2 r_{1}^{\prime} K^{\prime} \sqrt{-1}}{n} .
\end{gathered}
$$


44 Rev. B. Bronwin on Jacobi's General Transformation

The third form, therefore, reduces to the first.

Also,

$\sin ^{2} a m(2 \omega)=\sin ^{2} a m\left(2 \omega-2 K^{\prime} \sqrt{-1}\right)=\sin ^{2} a m\left(2 \omega^{\prime}\right)$, if $\omega^{\prime}=\omega-K \sqrt{-1}$. Let

$$
\omega=\frac{(2 r+1) \mathrm{K}+\left(2 r^{\prime}+1\right) \mathrm{K}^{\prime} \sqrt{-1}}{n} \text {. }
$$

Then

$$
\begin{gathered}
\omega^{\prime}=\frac{(2 r+1) \mathrm{K}+\left(2 r^{\prime}-2 p+2\right) \mathrm{K}^{\prime} \sqrt{-1}}{n} \\
=\frac{(2 r+1) \mathrm{K}+2 r_{1}^{\prime} \mathrm{K}^{\prime} \sqrt{\overline{-1}}}{n},
\end{gathered}
$$

which reduces the fourth form to the first. For, from what has been demonstrated, it follows that

$$
\begin{aligned}
& \sin ^{2} a m(2 m \omega)=f\left\{\sin ^{2} a m(2 \omega)\right\}=f\left\{\sin ^{2} a m\left(2 \omega^{\prime}\right)\right\} \\
& =\sin ^{2} a m\left(2 m \omega^{\prime}\right) \sin ^{2} \operatorname{co} a m(2 m \omega)=f_{1}\left\{\sin ^{2} a m(2 \omega)\right\} \\
& =f_{1}\left\{\sin ^{2} a m\left(2 \omega^{\prime}\right)\right\}=\sin ^{2} \operatorname{coa} a\left(2 m \omega^{\prime}\right),
\end{aligned}
$$

where $m$ is any integer, $f$ and $f_{1}$ denote certain functions, $\omega$ stands for one of the last three forms of this quantity, and $\omega^{\prime}$ for the first form. To these we may add

$$
\begin{aligned}
& \cos ^{2} a m(2 m \omega)=1-\sin ^{2} a m(2 m \omega)=1-\sin ^{2} a m\left(2 m \omega^{\prime}\right) \\
& =\cos ^{2} a m\left(2 m \omega^{\prime}\right) .
\end{aligned}
$$

Jacobi's transformation reduced is

$$
\begin{aligned}
& \int_{0}^{y} \frac{d y}{\sqrt{\left(1-y^{2}\right)\left(1-\lambda^{2} y^{2}\right)}}=\frac{1}{\mathrm{M}} \int_{0}^{x} \frac{d x}{\sqrt{\left(1-x^{2}\right)\left(1-k^{2} x^{2}\right)}} \\
& \left.\begin{array}{c}
y=\frac{x}{\mathrm{M}} \frac{1-\frac{x}{\mathrm{~s}^{2} a(2 \omega)}}{1-k^{2} x^{2} \mathrm{~s}^{2} a(2 \omega)} \cdot \frac{1-\frac{x}{\mathrm{~s}^{2} a(4 \omega)}}{1-k^{2} x^{2} \mathrm{~s}^{2} a(4 \omega)} \\
\ldots \ldots \ldots . \frac{1-\frac{x^{2}}{\mathrm{~s}^{2} a(n-1) \omega}}{1-k^{2} x^{2} \mathrm{~s}^{2} a(n-1) \omega}
\end{array}\right\} .
\end{aligned}
$$
where $\omega$ may have any of the four forms. Let $\omega$ again denote any of the last three forms, $\omega^{\prime}$ the first. In (1.) and in the expressions of $M$ and $\lambda$, we may charige $\omega$ into $\omega^{\prime}$, and the values of all the coefficients and all the constants will remain unchanged. There is then but one transformation.

We easily transform (1.) into

$$
y=\frac{\mathrm{s} a \cdot u \mathrm{~s} a\left(u+2 \omega^{\prime}\right) \ldots \ldots \mathrm{s} a\left(u+(2 n-2) \omega^{\prime}\right)}{\mathrm{s} a \cdot \omega^{\prime} \mathrm{s} a\left(3 \omega^{\prime}\right) \ldots \ldots \mathrm{s} a(2 n-1) \omega^{\prime}} .
$$


Make $u=0,2 \omega^{\prime}, 4 w^{\prime}, \&$ c. ; and we have $y=0$. Again, make $u=\omega^{\prime}, 3 \omega^{\prime}, 5 \omega^{\prime}, 8 c$.; and we have $y= \pm 1$. For the other forms of $\omega$ we have

$$
y=\frac{\mathrm{s} a \cdot u \mathrm{~s} a(u+2 \omega) \ldots \ldots \mathrm{s} a(u+(2 n-2) \omega)}{\mathrm{s} a(\mathrm{~K}-2 \omega) \mathrm{s} a(\mathrm{~K}-4 \omega) \ldots \ldots \mathrm{s} a(\mathrm{~K}-(2 n-2) \omega)} .
$$

When $u=K, K-2 \omega, \& c$, this gives $y= \pm 1$. But which of these values falls between $u=0, u=2 \omega$, or between $u=0$, $u=2 \omega^{\prime}$, we cannot tell. We ought, however, in good factorial formulæ to know. And when $u=\omega^{\prime}, 3 \omega^{\prime}, \&$ c., this must give $y= \pm 1$; because (3.) must give the same value of $y$ that (2.) does. But when we put for $\omega^{\prime}$ its value in $\omega$, this does not appear; nor can we reduce the result, except for the second form of $\omega$, without eliminating $\omega$, which would be equivalent to reducing (3.) to (2.) But all these things ought to be apparent in the face of factorial formula. Other faults might be pointed out; and we might point out similar fanlts in the expression of the value of $\sqrt{1-y^{2}}$. But I shall not dwell upon the subject. If the last three forms of $\omega$ do not render the valnes of $y$ erroneous, they render the formulæ faulty. 'They are unnatural; they reduce to the first form, and there is but one transformation.

If Mr. Cayley had proved in his paper of November 1844 all that he wished, it would not follow that the different forms of $\omega$ would give really distinct transformations. But his formula (6.) is not Jacobi's. To agree with his, the first member should be $\phi_{1}\left(\frac{u}{M}\right)$, the denominator of the second

$$
\phi(\mathrm{K}-2 \omega) \Phi(\mathrm{K}-4 \omega) \ldots \phi(\mathrm{K}-(2 n-2) \omega) \text {. }
$$

He might however, by a suitable modification of (5.), have arrived at Jacobi's result. But his proof of the possibility of

$$
m \mathrm{~K}+m^{\prime} \mathrm{K}^{\prime} \sqrt{-1}+\cdot \theta=\mu \mathrm{H}+\mu^{\prime} \mathrm{H}^{\prime} \sqrt{-1}
$$

is not to me satisfactory. It is unnecessary to state my objections, because he has not shown the possibility of $2 m \mathrm{~K}+\left(2 m^{\prime}+1\right) \mathrm{K}^{\prime} \sqrt{-1}+2 r \theta=2 \mu \mathrm{H}+\left(2 \mu^{\prime}+1\right) \mathrm{H}^{\prime} \sqrt{-1}$.

The omission of this appears to me very strange. Ot course the omission renders his paper perfectly nugatory. For the possibility of this equation does not follow from that of the former, supposing that to be fairly proved. If he had shown the possibility of this last, I believe he would have found it necessary to modify a little the form of some of the quantities $a, b, \& c$. Moreover, if he had transformed the factorial values of $f(u), \mathrm{F}(u)$ (I use here Abel's symbols), he might have found it necessary to modify them still further. In a com- 
plete proof, such as $\mathrm{Mr}$. Cayley professes his to be, all these things ought to have been done. And I heartily wish he had done them, and done them rightly, for the sake of the result.

Mr. Cayley thinks that his paper just referred to will lead to the complete determination of $\mathrm{H}$ and $\mathrm{H}^{\prime}$. I think so too; and I say that $H=\frac{\omega^{\prime}}{M}$, and that this follows from (2.) of this paper. But from the last three forms of os I cannot determine H. And if I could, it is easy to see from (3.) that they would give different values to this quantity; another proof of the faulty nature of these forms. For the transformation being but one, $\mathrm{H}$ ought to have but one value. I suppose the different forms of $w$ derived from one another, as in this paper.

With regard to Mr. Cayley's last paper, I have to observe that I had made trial of the form $w=\frac{K^{\prime} \sqrt{-1}}{3}$, and did not make it to be a transformation. But on going over the subject again, I find I somewhere made a mistake. It is complementary however, and makes no part of the direct transformation, although somehow strangely derived from it, without the process by which the complementary is derived from the direct one. I pass over the rest of this paper, because it would be an endless and useless task to discuss every minute particular.

Gunthwaite Hall, May 13, 1845.

XIII. On Fresnel's Theory of Diffraction. By R. Moon, M.A., Fellow of Qucen's College, Cambridge, and of the Cambridge Philosophical Socicty.

[Continued from vol. xxvi. p.94.]

T $N$ a paper which appeared in a recent Number of this 1 Journal (see vol. xxvi. p. 89), I endeavoured to point out some remarkable errors in Fresnel's investigation of the fringes produced by an opake body illuminated from a single point; and in particular I endeavoured to stigmatize an erroneous principle of approximation adopted by Fresnel, and which I then stated, and now assert to be such, as to vitiate every investigation of that ingenious person in this department of optics. I thence proceeded to show that Fresnel's mode of investigation, when properly conducted, leads to conclusions very different from what he supposes, and such in fact as are completely at variance with the observed phænomena which he wholly fails to account for ; and I further drew the conclusion, that the principle of small waves emanating from the 